\title{
Research Progress of Unilateral Biportal Endoscopic Spinal Surgery in Lumbar Spine
}

\author{
Jun-Long Hou ${ }^{1}$, Shaojie $\mathrm{He}^{1}$, Zhi-Zhong $\mathrm{Li}^{1}$
}

${ }^{1}$ First Affiliated Hospital of Jinan University, No. 613 Huangpudadao, Tianhe District, Guangzhou 510632

Abstract: Percutaneous spinal endoscopy is rapidly popularized in China because of its advantages of small surgical side injury and fast postoperative recovery. However, this technology is usually operated in a single channel, which has the disadvantages of limited surgical field of vision, small range of instrument activities and low work efficiency. In unilateral double channel spinal endoscopic surgery, one channel is equipped with endoscope and flushing function at the same time, and the other channel is used for the operation of surgical instruments. Compared with the previous single channel, it has the advantages of larger surgical field of vision, more flexible instrument operation, shorter learning curve, the use of traditional open surgical instruments familiar to the operator, and fewer intraoperative fluoroscopy times. This paper analyzes the current situation and clinical value of its clinical application in lumbar diseases.

Keywords: Lumbar Spine, Endoscopy, Surgery, Minimally Invasive, Literature Review

\section{Introduction:}

Minimally invasive spine surgery is a research hotspot in spine surgery at present. Its purpose is to provide the same treatment effect as traditional open surgery, reduce the side injury caused by the surgical approach as far as possible, and make the patients return to normal life earlier. ${ }^{[1]}$ In recent years, unilateral biportal endoscopic spinal surgery has made rapid development in the continuous exploration and improvement of multinational spine surgeons represented by Korean doctors. It has been able to perform operations such as resection of complex intervertebral disc herniation, unilateral approach, bilateral decompression and interbody fusion, and has improved the intraoperative field of vision and expanded the scope of work, It has been gradually applied in the clinical treatment of a variety of spinal surgical diseases, and achieved ideal clinical efficacy. ${ }^{[2-3]}$ This paper analyzes the clinical application status and clinical value of unilateral biportal endoscopic spinal surgery in spinal diseases.

\section{Development of unilateral biportal endoscopic spinal surgery:}

UBE is an improved minimally invasive spinal endoscopy technique, which refers to spinal endoscopic surgery through two channels on one side. One channel of the dual channel spinal endoscopy places the endoscope and has the flushing function at the same time, and the other channel is used for the operation of surgical instruments. Basic surgical instruments include Kerrison bite forceps, $0^{\circ}$ or $30^{\circ}$ arthroscopy, bipolar radiofrequency, grinding drill, nerve stripping ion, bone chisel, etc.. Conventional instruments can be operated freely from multiple angles through the working channel. ${ }^{[4]}$ Surgery can be roughly divided into inter laminar approach and trans foraminal approach, and then take corresponding operations according to different diseases.

In the 1980 s, forst ${ }^{[5]}$ first used arthroscopy in the treatment of lumbar disc herniation under direct vision, which improved the safety and effectiveness of the operation and laid a foundation for the development of endoscopic discectomy. Then kambin et al began to try to use arthroscopy for lumbar discectomy and gradually applied it to the treatment of lumbar spinal stenosis $^{[6]}$. In 1996, De Antoni and others improved the technology so that it can be completed by one person ${ }^{[7]}$. Ube technology was proposed and reported for the first time. By establishing two channels, the field of vision under the mirror is wider and the operation is more flexible. In 1999, Yeung et al. ${ }^{[8]}$ Developed the third generation spinal endoscopy system (yes) technology. The intraoperative endoscope enters the intervertebral disc through the "kambin triangle" area of the intervertebral disc ${ }^{[9-10]}$. Since then, percutaneous endoscopic discectomy (petd) through the intervertebral foramen has been widely used. In 2006, Choi et al. Reported for the first time that percutaneous endoscopic discectomy via intervertebral space approach is suitable for patients with L5 / S1 segmental lesions and anatomical features such as high iliac crest, sacral lumbarization and transverse process hypertrophy ${ }^{[11]}$. In 2013, Soliman and others systematically reported that 43 patients with lumbar disc herniation underwent arthroscopic assisted percutaneous unilateral double channel discectomy, and the surgical effect was satisfactory ${ }^{[12]}$. Unilateral biportal endoscopic spinal surgery has the advantages of clear intraoperative field of vision, conventional surgical instruments, wide operation range, contralateral recess stenosis and good decompression.
Application of UBE technique in lumbar diseases Application of in lumbar disc herniation
In 2016, Eun et al. Performed UBE submedullary nucleus enucleation in 11 patients with LDH. After 14 months of follow-up, the efficacy was analyzed by comparing the preoperative and postoperative VAS scores and dysfunction index (ODI) scores of the waist and legs. The results showed that the preoperative vas and ODI scores of the legs were significantly lower 
than those before operation ${ }^{[13]}$. Kim et al reported that patients with single segment lumbar disc herniation were treated with Ube technology and microscopic discectomy (MD). The intraoperative bleeding and hospital stay in Ube group were lower than those in MD group; The incidence of postoperative complications, VAS score and ODI score were similar in the two groups ${ }^{[14]}$. Choi et al reported that 68 patients with degenerative lumbar diseases were treated with Ube / Bess technology. The average operation time of single segment lumbar disc herniation was 68.2 min and the incidence of complications was $10.3 \%^{[15]}$. Ube technology is an improvement of single channel spinal endoscope technology. The independent working channel allows the operator to operate basic orthopedic instruments, which is conducive to shortening the operation time and improving the operation efficiency. It has the same decompression effect as MD, less damage to soft tissue, less intraoperative bleeding, short postoperative recovery period and low incidence of perioperative complications. It can be seen that Ube technology is expected to be popularized as an alternative to open surgery in the treatment of recurrent disc herniation in the future.

\section{Application of in lumbar spinal stenosis}

Lumbar spinal stenosis is a clinical syndrome, which refers to the stenosis of spinal canal, nerve root canal and intervertebral foramen caused by any reason other than the independent disease leading to lumbar spinal stenosis, and the syndrome of cauda equina nerve or nerve root compression ${ }^{[16]}$. Soliman reported a prospective study of 104 patients with lumbar spinal stenosis treated with Ube / Bess technology. The average operation time and estimated bleeding volume per segment were $62.8 \mathrm{~min}$ and $60 \mathrm{ml}$, respectively; All patients were followed up for an average of 28 months, of which 6 cases had dural sac tear. The satisfactory rate of postoperative effect was $87 \%^{[17]}$. Hwa et al reported a preliminary study on the application of UBE / Bess technology in 58 patients with single segment lumbar spinal stenosis. The average age of the patients was 63.4 years, the average follow-up was 13.8 months, and the average operation time was 68.9 minutes; Related complications occurred in 8 cases, which were improved after symptomatic treatment; The satisfactory rate of postoperative efficacy was $81 \%^{[18]}$. Torudom et al reported 30 cases of lumbar spinal stenosis treated with Ube / Bess Technology (25 cases of single segment and 5 cases of double segment). The average operation time of single segment and double segment were $98.3 \mathrm{~min}$ and $170.4 \mathrm{~min}$ respectively. After follow-up for more than 2 years, the incidence of complications was $6.6 \%$. The postoperative low back and leg pain was significantly relieved, and the curative effect satisfaction rate was $83.3 \%{ }^{[19]}$. Kim et al. Used Ube / Bess technique to decompress 105 patients with lumbar spinal stenosis. The average age of the patients was 71.2 years, the average follow-up was 14 months, and the average operation time was 53 minutes. The postoperative ODI score and VAS score were significantly improved compared with those before operation, and the satisfaction rate of postoperative curative effect was $88 \%^{[20]}$. HEO et al. Conducted a comparative study on the efficacy of three surgical methods in the treatment of lumbar spinal stenosis, namely, microsurgical decompression group (33 cases), dual channel spinal canal decompression group (37 cases) and single channel endoscopic spinal canal decompression group (27 cases). The surgical efficacy was evaluated by comparing the operation time, postoperative complications, preoperative and postoperative low back and leg pain VAS score, ODI index and postoperative spinal canal expansion area. After an average follow-up of $(12.5 \pm 3.3)$ months, the results showed that there was no significant difference in operation time, complications, VAS score and ODI score of low back and leg pain; However, the double channel operation group showed advantages in the expansion of spinal canal area, the preservation of articular process and the VAS score of low back pain on the first day after operation ${ }^{[21]}$. Research shows that Ube technology has at least the same clinical efficacy as open microscope surgery and single channel endoscopic surgery, and further confirms the effectiveness and safety of UBE technology in the treatment of lumbar spinal stenosis. It is believed that Ube technology will be gradually popularized in the near future.

\section{Application of in spinal interbody fusion}

UBE / BESS technology can be used to treat patients with vertebral instability who need interbody fusion and internal fixation. At present, the reported unilateral double channel spinal endoscopic interbody fusion (ULIF) mostly adopts the posterior approach. Under the endoscope, the ipsilateral decompression, contralateral exploration and complete removal of the intervertebral disc and cartilage endplate are completed, and then the fusion cage is placed under the direct vision of the endoscope, and the pedicle screws are placed through the original double channel incision. Heo et al reported 69 cases of single segment interbody fusion using UBE/BESS technology, including 51 cases of degenerative lumbar spondylolisthesis, 9 cases of isthmus with lumbar spondylolisthesis, 6 cases of central spinal canal stenosis with instability, and 3 cases of central spinal canal stenosis with intervertebral foramen stenosis. The postoperative curative effect was satisfactory, and the incidence of complications was $7.2 \%$, including 2 cases of dural tear and 3 cases of epidural hematoma, All patients were cured by non-surgical treatment; The rate of interbody fusion has not been reported ${ }^{[22]}$. Kim et al. Used Ube / Bess technology to perform interbody fusion in 14 patients, including 4 cases of degenerative lumbar spondylolisthesis, 2 cases of pedicle isthmus with lumbar spondylolisthesis, 8 cases of central spinal canal stenosis with intervertebral foramen stenosis. The incidence of postoperative complications was $14.3 \%$, including 1 case of dural tear and 1 case of L5 nerve root numbness, which improved after non-surgical 
treatment ${ }^{[23]}$. Park et al. Compared and studied the clinical efficacy of ulif (71 cases) and conventional PLIF (70 cases) in the treatment of lumbar degenerative diseases. The results showed that there was no significant difference between the two surgical methods in preoperative and postoperative leg VAS score, ODI score, surgical fusion rate and postoperative complications, but the waist and back VAS score of ulif group was significantly lower than that of PLIF group within 1 week after operation, In terms of operation time, ulif Group [(158.2 \pm 26.7$)$ min] was significantly higher than PLIF Group $[(136.6 \pm 21.5) \mathrm{min}]^{[24]}$. At present, the short-term follow-up results show that the use of UBE / Bess technology for interbody fusion can improve the postoperative symptoms and achieve good results, but the recurrence rate and postoperative biomechanical stability of lumbar spine still need long-term follow-up study. At present, the operation time of UBE is generally longer than that of routine operation, but it is believed that with the growth of doctors and technical improvement, Ube technology will shine in the treatment of various spinal diseases in the future.

\section{Summary and Prospect}

Based on the existing clinical reports, we believe that Ube has the following potential advantages: (1) Endoscopy and operating instruments do not have to restrict each other, the scope of operation field is larger, the structure identification is more convenient, and the safety of operation is increased; (2) The instrument operation is more flexible. It has prominent advantages in unilateral approach and bilateral decompression, and can obtain a larger range of surgical activities; (3) The visual field and operation are closer to open surgery, which is more conducive for beginners to master, and the learning curve is relatively flat; (4) Surgical instruments are ready-made and relatively cheap. Traditional open surgical instruments can be used, and the operator is more familiar with them; (5) Intraoperative fluoroscopy was less than PELD, and the radiation dose to surgeons and patients was less ${ }^{[25]}$.Ube / Bess technique is usually suitable for diseases difficult to be solved by petd, including central spinal canal stenosis with bilateral lateral recess stenosis, lumbar nerve root canal stenosis and medium to large intervertebral disc herniation. The current research results suggest that the efficacy of UBE technology in the treatment of lumbar degenerative diseases is more accurate, but compared with conventional open surgery and single channel endoscopy, its safety and efficacy are still lack of high-level evidence support, so it needs to be further evaluated and verified. Therefore, a large sample prospective randomized study and long-term follow-up are needed to comprehensively evaluate the medium and long-term efficacy of this technique. With the rapid development and continuous innovation of UBE technology, more spine surgeons will master this technology and conduct high-quality relevant clinical research in the future, so as to provide further evidence-based medicine evidence for Ube endoscopic technology to better serve the majority of patients.

\section{Reference}

1. Celik SE, Celik S, Göksu K, et al. Microdecompressive la minatomy with a 5-year follow-up period for severe lumba r spinal stenosis[J]. J Spinal Disord Tech, 2010, 23(4): 22 9-235. DOI:10.1097/BSD.0b013e3181a3d889.

2. Hwa Eum J, Hwa Heo D, Son SK, et al. Percutaneous bi portal endoscopic decompression for lumbar spinal stenosis: a technical note and preliminary clinical results $[\mathrm{J}]$. J Neur osurg Spine,2016,24(4):602-607.DOI:10.3171/2015.7.SPINE1 5304.

3. Choi DJ, Jung JT, Lee SJ, et al. Biportal endoscopic spina 1 surgery for recurrent lumbar disc herniations[J]. Clin Ort hop Surg,2016,8(3):325-329.DOI:10.4055/cios.2016.8.3.325.

4. Choi CM, Chung JT, Lee SJ, et al. How I do it? Biportal endoscopic spinal surgery (BESS) for treatment of lumbar spinal stenosis[J].Acta Neurochir (Wien),2016,158(3):459-4 63. DOI:10.1007/s00701-015-2670-7.

5. Forst R, Hausmann B. Nucleoscopy-a new examination $t$ echnique [ J ] . Arch Orthop Trauma Surg , 1983, 101 ( 3 ) : 219-221

6. Kambin P. Diagnostic and therapeutic spinal arthroscopy[ J ] Neurosurg Clin N Am, 1996,7 ( 1 ): 65-76.

7. De Antoni DJ, Claro ML, Poehling GG, et al. Translami nar lumbar epidural endoscopy : anatomy, technique, an d indications [ J ] . Arthroscopy , 1996,12 ( 3 ): 330-334.

8. Yeung AT. Minimally invasive disc surgery with the Yeun $\mathrm{g}$ endoscopic spine system( YESS ) $\mathrm{J}$ ]. Surg Technol Int , $1999,8: 267-277$

9. Kambin P, Casey K, O’Brien E, et al. Transforaminal art hroscopic decompression of lateral recess stenosis $[\mathrm{J}]$. J Neurosurg , 1996 , 84 ( 3 ): 462-467.

10. Kambin P, O'Brien E, Zhou L, et al. Arthroscopic micro discectomy and selective fragmentectomy [ J ] . Clin Orthop Relat Res , 1998 ( 347 ): 150-167

11. Choi G, Lee SH, Raiturker PP, et al. Percutaneous endos copic interlaminar discectomy for intracanalicular disc herni ations at L5-S1 using a rigid working channel endoscope [ J ] . Neurosurgery , 2006 , 58 ( 1 Suppl ): ONS59-ONS6 8 .

12. Soliman HM. Irrigation endoscopic discectomy: a novel pe rcutaneous approach for lumbar disc prolapse[J]. Eur Spine J, 2013, 22(5): 1037- 1044 .

13. Eun SS, Eum JH, Lee SH, Sabal LA. Biportal endoscopic lumbar decompression for lumbar disk herniation and spin al canal stenosis: A technical note[J]. J Neurol Surg A Ce nt Eur Neurosurg, 2017,78(4):390-396.

14. Kim SK, Kang SS, Hong YH, et al. Clinical comparison of unilateral biportal endoscopic technique versus open mic rodiscectomy for single-level lumbar discectomy: a multice nter, retrospective analysis[J] J Orthop Surg Res, 2018,13(1) :22.

15. Choi DJ, Choi CM , Jung JT, et al. Learning curve assoc iated with complications in biportal endoscopic spinal surg ery : challenges and strategies [ J ]. Asian Spine J , 2016, $10(4): 624-629$.

16. Kim JE, Choi DJ, Park EJ. Clinical and radiological outco mes of foraminal decompression using unilateral biportal e ndoscopic spine surgery for lumbar foraminal stenosis. Cli n Orthop Surg, 2018, 10 (4) :439 - 447.

17. Soliman HM. Irrigation endoscopic decompressive laminoto my. A new endoscopic approach for spinal stenosis decom pression [ J ] . Spine J , 2015 , 15 ( 10 ): 2282-2289.

18. Hwa Eum J , Hwa Heo D, Son SK, et al. Percutaneous b iportal endoscopic decompression for lumbar spinal stenosis : a technical note and preliminary clinical results $[\mathrm{J}]$. J Neurosurg Spine , 2016 , 24 ( 4 ): 602-607.

19. Torudom Y, Dilokhuttakarn T. Two portal percutaneous en doscopic decompression for lumbar spinal stenosis : preli minary study [ J ] . Asian Spine J , 2016 , 10 ( 2 ): 335-3 42

20. Kim JE, Choi DJ. Unilateral biportal endoscopic decompre 
ssion by $30^{\circ}$ endoscopy in lumbar spinal stenosis : technic al note and preliminary report [ J ] . J Orthop , 2018, 15 ( 2 ) : 366-371.

21. Heo DH, Lee DC, Park CK. Comparative analysis of thre e types of minimally invasive decompressive surgery for 1 umbar central stenosis: biportal endoscopy, uniportal endos copy, and microsurgery[J]. Neurosurg Focus, 2019, 46(5): E9.

22. Heo DH, Son SK, Eum JH, et al. Fully endoscopic lumb ar interbody fusion using a percutaneous unilateral biportal endoscopic technique : technical note and preliminary cli nical results [ J ] . Neurosurg Focus, 2017, 43 ( 2) : E8.

23. Kim JE, Choi DJ. Biportal endoscopic transforaminal lumb ar interbody fusion with arthroscopy [ $\mathrm{J}]$. Clin Orthop Sur g, 2018, $10(2): 248-252$.

24. Park MK, Park SA, Son SK, et al.Clinical and radiologica 1 outcomes of unilateral biportal endoscopic lumbar interbo dy fusion (ULIF) compared with conventional posterior lu mbar interbody fusion (PLIF): 1-year follow-up[J]. Neurosu rg Rev, 2019,42(3):753-761.

25. Eun SS, Eum JH, Lee SH, et al. Biportal endoscopic lum bar decompression for lumbar disk herniation and spinal $\mathrm{c}$ anal stenosis: a technical note[J]. J Neurol Surg A Cent E ur Neurosurg,2017,78(4):390-396.DOI:10.1055/s-0036-15921 57. 